\title{
Safety Analysis of the GEM Detector Magnet Conductor
}

\author{
M.A. Ferri and W.V. Hassenzahl
}

August 27, 1993

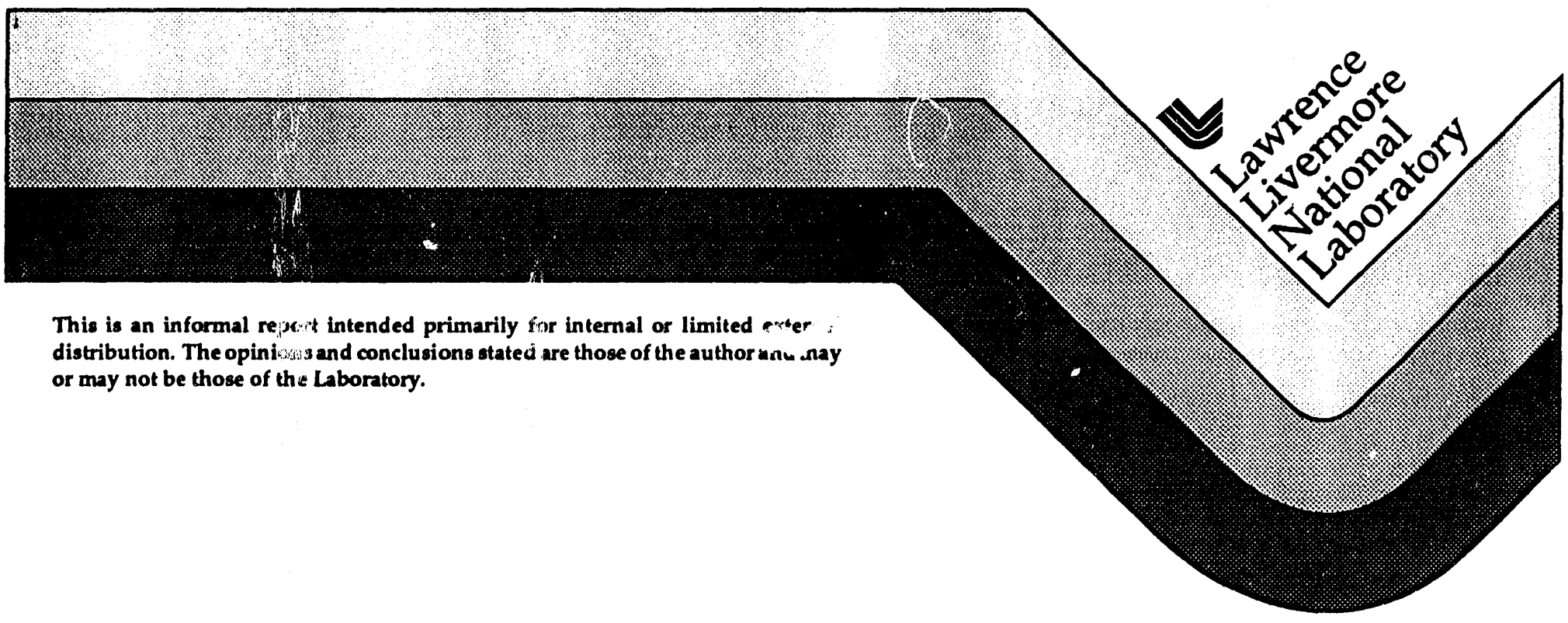

This is an informal re intended primarily for internal or limited arer distribution. The opinisus and conclusions stated are those of the author an nay or may not be those of the Laboratory. 


\section{DISCLAIMER}

This document was prepared as an account of work sponsored by an agency of the United States Government. Neither the United States Government nor the University of California nor any of their employees, makes any warranty, express or implied, or 2ssumes any legal liability or responsibility for the accuracy, completeness, or usefulness of any information, apparatus, product, or process disclosed, or represents that its use would not infringe privately owned rights. Reference herein to any specific commercial products, process, or service by trade name, trademark, manufacturer, or otherwise, does not necessarily constitute or imply its endorsement, recommendation, or favoring by the United States Government or the University of California. The views and opinions of authors expressed herein do not necessarily state or reflect those of the United States Government or the University of California, and shall not be used for advertising or product endorsement purposes.

This report has been reproduced directly from the best available copy.

Available to DOE and DOE contractors from the Office of Scientific and Technical Information

P.O. Box 62, Oak Ridge, TN 37831

Prices a vailable from (615) 576-8401, FTS 626-8401

Available to the public from the

National Technical Information Service

U.S. Department of Commerce

5285 Port Royal Rd.,

Springfield, VA 2216 


\title{
Safety Analysis of the GEM Detector Magnet Conductor
}

\author{
M. A. Ferri*, W. V. Hassenzahl \\ Lawrence Livermore National Laboratory \\ Livermore, CA 94551
}

\begin{abstract}
The safety of the GEM Detector Magnet is analyzed using a computational model to determine current sharing between the cabled conductor and the external aluminum stabilizer. The model includes inductive and transvers: conductive effects due to the geometries of the coil and the conductor. A conservative analysis indicates a peak conductor hotspot temperature of $\sim 50 \mathrm{~K}$ at two seconds after the initiation of quench. After this time, additional heating is limited because most of the current in the normal zone region is carried by the aluminum stabilizer and an external protection circuit should have begun to diminish the total current. The analysis shows that conductor safety requires adequate transverse conductivity between the cable and the aluminum stabilizer. The calculated transverse conductance of the GEM conductor, $1 \times 10^{7} \mathrm{mho} / \mathrm{m}$, is at least 100 times greater than the minimum value necessary to limit the hotspot temperature to $\sim 50 \mathrm{~K}$ after two seconds. This report describes the results of calculations based on a realistic assumption of GEM conductor performance during a quench.
\end{abstract}

\footnotetext{
${ }^{*}$ Permanent address: Plasma Fusion Center, Massachusetts Institute of Technology, Cambridge MA 02139
} 


\section{INTRODUCTION}

This paper describes an analysis by the Applied Superconductivity Group at Lawrence Livermore National Laboratory to determine the safety characteristics of the conductor for the Superconducting Super-Collider GEM Detector Magnet. The analysis is concerned solely with the safety of the conductor as defined by the peak temperature reached during the initial few seconds of a quench. The issue of magnet protection on longer time scales, including energy removal, voltages, etc., is not addressed. This limited scope is based on an estimate that the conductor temperature would reach $150 \mathrm{~K}$ in one second if the operating current of $50 \mathrm{kA}$ remained in the superconductor and copper stabilizer without adequate transfer to the aluminum sheath. Since this temperature already surpasses the prescribed limit of $100 K_{1}^{1}$ it is necessary to accurately describe the transient currents in the conductor on short time scales in order to correctly judge the safety of the conductor design.

A previous analysis of a quench in the GEM magnet evaluated a scenario in which current was transferred from the conductor to the aluminum sheath at the ends of each $1140 \mathrm{~m}$ long coil section. ${ }^{2}$ Although reference [2] indicated a high margin of safety for the magnet, it did not include inductive effects associated with either conductor or coil geometry. It also did not incorporate transverse conductivity as an influence in current transfer, as was evaluated in reference [3]. During a design review of the GEM project in May 1993, reviewers questioned the analysis of magnet safety. Since this issue had been addressed in earlier reviews, the GEM magnet designers were not present to substantiate their results. The review committee therefore suggested that the independent analysis presented here would be appropriate to assure the successful design and operation of the magnet.

The GEM magnet is approximately $20 \mathrm{~m}$ in diameter and $31 \mathrm{~m}$ long. It is divided into 24 coil segments composed of $1140 \mathrm{~m}$ of conductor each. The conductor is characterized by a large external aluminum stabilizer, the "sheath," around a cable-in-conduit $\mathrm{Nb}-\mathrm{Ti}$ superconductor, as shown in the cross-section in Fig. 1. Table 1 lists a set of parameters of the conductor, from references [2] and [3], which were used in all calculations.

To determine the conductor's short-term temperature response to a quench, it is necessary to calculate the joule heating resulting from current in 
the conductor as a function of time. In this analysis, the development and solution of the correct electrical circuit equations, including inductive and transverse conductive effects, are used to study the temperature rise during quench in the GEM magnet. The resulting peak temperature of $\sim 50 \mathrm{~K}$ after 2 seconds, when much of the current has transferred to the aluminum, is determined to be acceptable.

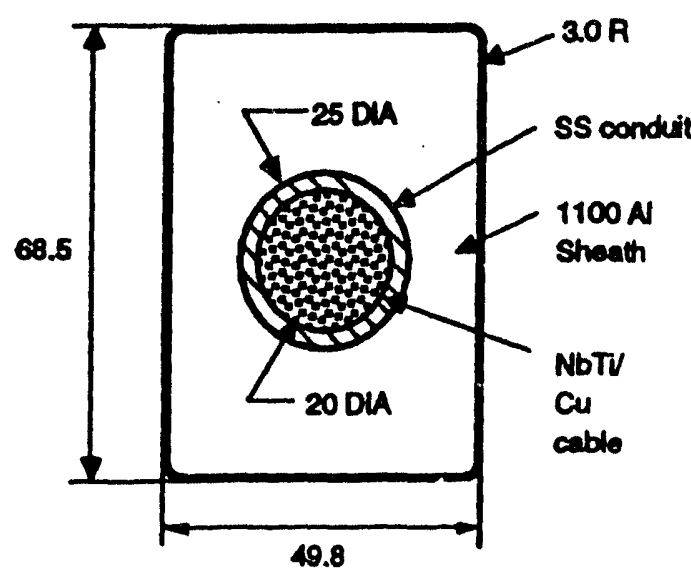

Figure 1. Cross section showing the geometry of the GEM conductor. Dimensions are in $\mathrm{mm}$. (From reference [1].)

\begin{tabular}{|l|l|}
\hline Overall Width $(\mathrm{mm})$ & 45.10 \\
\hline Overall Height $(\mathrm{mm})$ & 68.50 \\
\hline Coil Section Length $(\mathrm{m})$ & 1140 \\
\hline Operating Current $(\mathrm{kA})$ & 50.2 \\
\hline Type of Superconductor & $\mathrm{Nb}-\mathrm{Ti}$ \\
\hline Cu : SC & $3: 1$ \\
\hline Copper RRR & 150 \\
\hline Strand Area $\left(\mathrm{mm}^{2}\right)$ & 188.3 \\
\hline Copper Area $\left(\mathrm{mm}^{2}\right)$ & 141.3 \\
\hline Helium Area $\left(\mathrm{mm}^{2}\right)$ & 116.4 \\
\hline Conduit Material & $304 \mathrm{SS}$ \\
\hline Conduit Thickness (mm) & 2.5 \\
\hline External Stabilizer (Sheath) Material & $1100 \mathrm{Al}$ \\
\hline External Stabilizer (Sheath) Area $\left(\mathrm{mm}^{2}\right)$ & 2920 \\
\hline
\end{tabular}

Table 1. Conductor parameters for the GEM magiet 


\section{SAFETY CONSIDERATIONS}

The peak temperature in a conductor during quench occurs at the center of the normal zone, where the quench is initiated. To determine if this "hotspot" temperature remains below the $100 \mathrm{~K}$ prescribed limit for the GEM magnet, it is useful to study an adiabatic volumetric heat balance at that location:

$$
\rho J^{2} d t=\gamma C_{p} d T
$$

where $\gamma_{p}$ is the volumetric average of the conductor specific heat capacity per unit volume; $\rho$ is the effective resistivity of the conductor; and $J$ is the current density in the conductor, which consists of the superconductor as well as the matrix. The adiabatic assumption ignores the presence of helium and is usually a good approximation since the characteristic time of a quench is usually less than one second. ${ }^{3}$ In any case, adiabaticity provides a conservative estimate.

Equation (1) can be rearranged and integrated to yield the relationship:

$$
\int_{0}^{t_{f}} I_{c o n d}^{2} d t=A_{c o n d}^{2} \int_{T_{b}}^{T_{f}} \frac{\not C_{p}}{\rho} d T=U\left(T_{f}\right) .
$$

$I_{\text {cond }}$ is the current flowing in the conductor; $A_{\text {cond }}$ refers to the conductor area; $T_{b}$ and $T_{f}$ are the initial and final temperatures respectively; and $t_{f}$ is the time associated with temperature $T_{f}$. The function $U\left(T_{f}\right)$ depends only on the properties of the conductor and, once calculated, can be used to determine the final temperature associated with any time-varying current in the conductor by using equation (2). Fig. 2 shows $U\left(T_{f}\right)$, calculated using numerical integration of existing correlations 5,6 for the thermal properties of the conductor, vs. final temperature, $T_{f}$, for an initial temperature, $T_{b}$, of $5 \mathrm{~K}$. From the plot, it is evident that to remain below the prescribed temperature limit $\left(T_{f}=100 \mathrm{~K}\right)$, the current must be limited to an integrated value of $I^{2} \mathrm{dt}$ approximately equal to $1.8 \times 10^{9} \mathrm{~A}^{2} \mathrm{~s}$. To achieve this limit requires two factors: 
1.) a rapid transfer of current from the resistive conductor zone to the aluminum sheath, and

2.) an external protection circuit to eventually bring the total current down to zero.

In this analysis, we consider the first of these two processes to be most critical since without current transfer the integrated current limit would already be surpassed within 1 second, a typical delay time before a protection circuit can be engaged. $\left(I_{o p} 2 \times 1 s=2.5 \times 10^{9} \mathrm{~A}^{2} \mathrm{~s} \rightarrow \mathrm{T}_{\mathrm{f}} \sim 150 \mathrm{~K}\right)$.

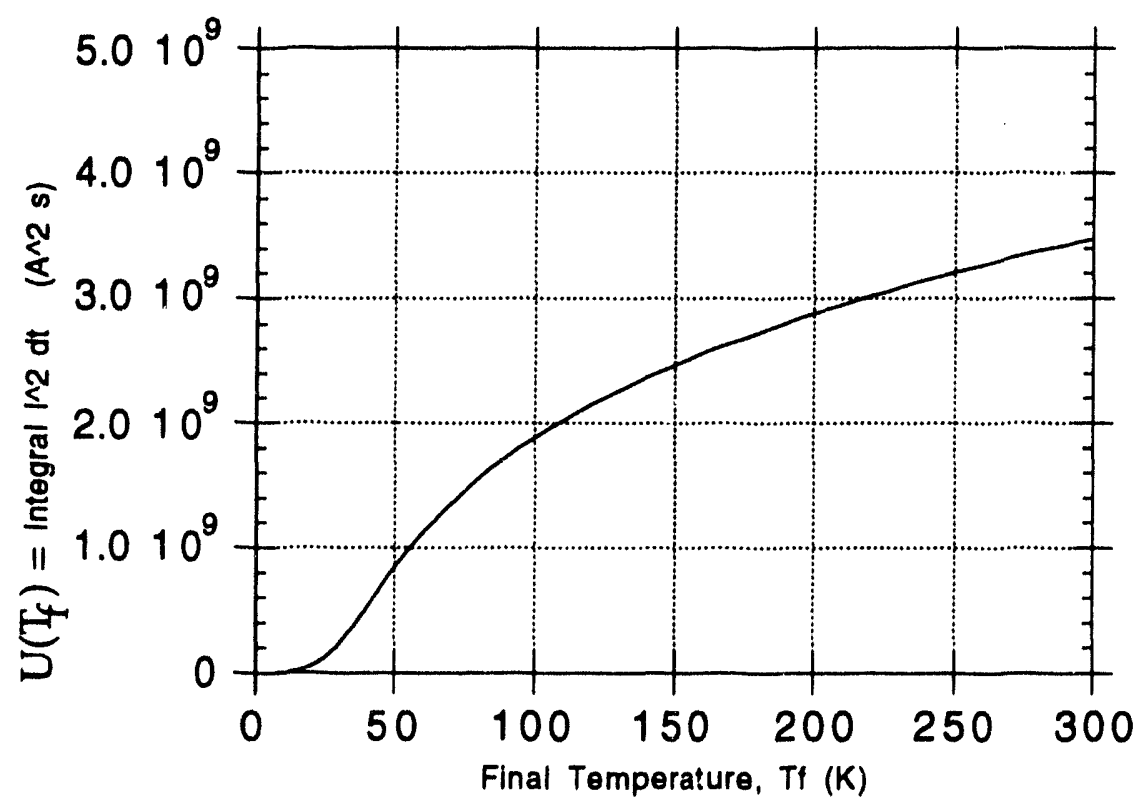

Figure 2. Time vs. Temperature curve for Gem Conductor. 


\section{CURRENT TRANSFER AT ENDS OF COIL SECTION}

The most conservative approach in analyzing the safety of the GEM conductor is to ignore the effects of transverse conductance between the conductor and the sheath, thus minimizing the amount of current which can be transferred out of the normal zone during quench. If the conductor safety characteristics resulting from this most conservative approach were to be acceptable, a more detailed analysis would not be needed. For this reason, this report begins by neglecting transverse conductance.

\subsection{Resistive Current Sharing Model For Transfer Only at Ends}

In a previous study, ${ }^{2}$ even though the transverse conductance was considered to be zero (the most conservative case), current transfer from superconductor to aluminum at the end of each coil section was adequate to keep the maximum temperature within allowed limits*. Current transfer was determined solely by resistive sharing (i.e., no inductive effects), giving the current in the conductor at any time to be:

$$
I_{c}=\frac{R_{a}}{R_{c}+R_{a}} I_{0}
$$

where $R_{c}$ and $R_{a}$ are integrated resistances over the entire length of the conductor and aluminum, respectively.

The quench code SARUMAN was used to self-consistently calculate the current in the conductor, from equation (3), during joule heating in the normal zone. ${ }^{2}$ Fig. 3 shows the resulting current in the conductor plotted vs. time. The decline in conductor current at $\sim 2.3$ seconds is due to a "quenchback effect" caused when joule heat uniformly generated along the sheath simultaneously quenched the entire length of superconductor. Uniform heating occurs because all the current transferred is forced to pass through the entire length of the aluminum sheath. The peak temperature in the conductor is shown in Fig. 4. Because of the quenchback effect, the conductor remains below the $100 \mathrm{~K}$ limit.

\footnotetext{
At the end of each coil section, the aluminum and conductor are considered to be in perfect electrical contact.
} 


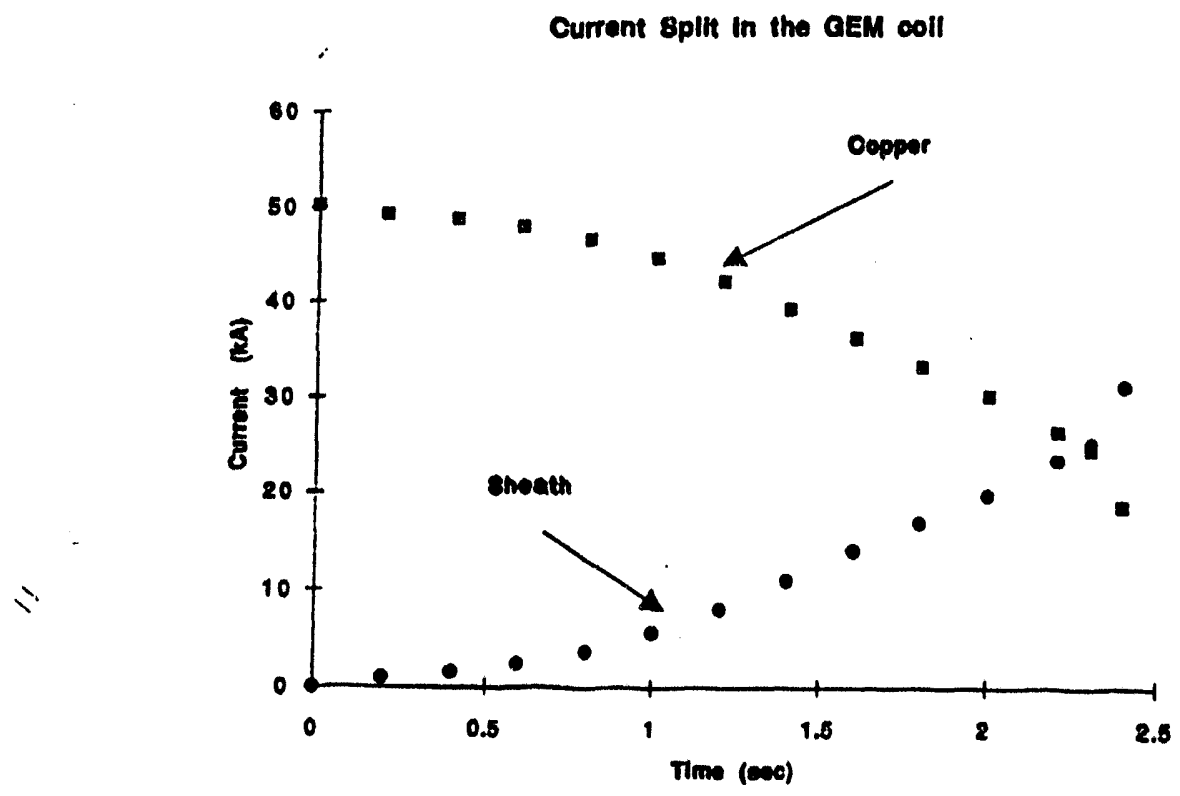

Figure 3. Currents in the Conductor and in the Sheath found in a previous study (reference [2]) using current transfer only at the ends of each coil section. The study notes that after 2.3 seconds, the current in the conductor falls dramatically due to the "quenchback effect."

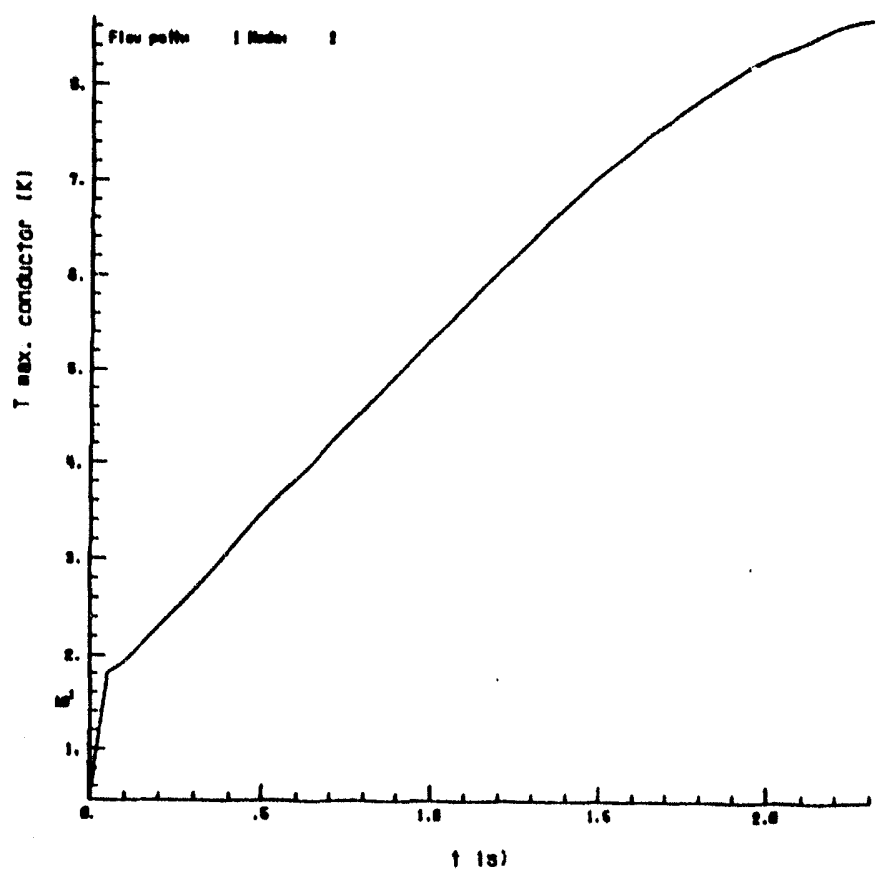

Figure 4. Hotspot temperature vs. time found in a previous study (reference [2]) consistent with currents shown in figure 3 . After 2.3 seconds, the temperature is $\sim 85 \mathrm{~K}$. 


\subsection{Resistive/Inductive Current Sharing Model for Transfer Only at Ends}

To begin the present study, the " $g_{t}$ equals zero" model of the previous study, discussed above, is briefly re-analyzed. This time, however, the timedependent effects of the mutual inductance between the conductor and aluminum are included. Fig. 5 displays a linear circuit approximately equivalent to the one formed by one coil section of GEM conductor with electrical contact only at the ends. To estimate the equivalent inductance of the coil section, the self inductance of this relevant circuit is calculated and the result then doubled to approximate the effects of the actual coil geometry, (i.e. adjacent turns of the coiled conductor approximately double the inductance of the linear model).

In the relevant linear circuit, for a current flow, I, through the conductor path, the flux penetrating the closed circuit, $\Phi$, can be approximated as:

$$
\Phi \approx B_{\text {ave }} A \quad, \quad B_{\text {ave }}=\frac{\mu_{o} I}{2 \pi r_{\text {ave }}}
$$

where $A=2 r_{a v e} L$ is the area of the circuit and $r_{a v e}$ is the distance from the conductor to the midpoint of area $\mathrm{A}$. The total mutual inductance is then (remembering the factor of two that approximates geometric effects):

$$
\ell \approx 2 \frac{\Phi}{I}=\frac{2 \mu_{o}}{\pi} L
$$

For a length, $\mathrm{L}$, of $1140 \mathrm{~m}$, this very rough estimate gives an equivalent inductance, $\ell$, on the order of $1 \mathrm{mH}$.

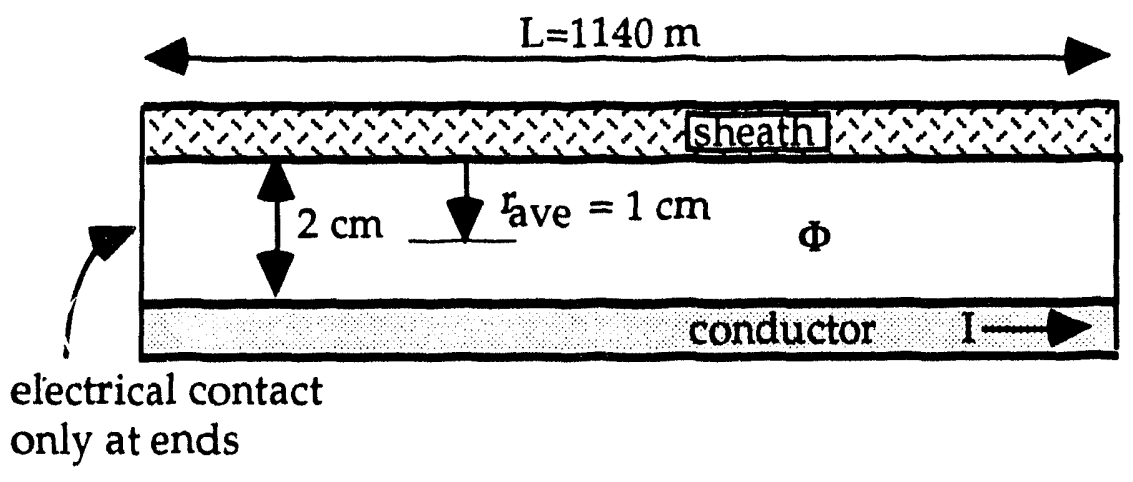

Figure 5. Approximately equivalent view of GEM conductor for purposes of calculating inductances (note different scales). 
For the low resistances of the conductor and aluminum at operating temperatures $(\sim 1 \mathrm{~m} \Omega$ each), an inductance of $1 \mathrm{mH}$ implies a current transfer time constant on the order of 1 second. Fig. 6 shows a rough estimate of the current in the conductor for a model that includes inductive effects but assumes a constant normal zone temperature of $10 \mathrm{~K}$ and a quench velocity of $20 \mathrm{~m} / \mathrm{s}$. Although very approximate, the results suggest that current transfer out of the conductor occurs significantly more slowly than was found previously, as shown in Fig. 3. This discrepancy casts doubt over the safety predictions of any analysis which does not include inductance. When inductive effects are included, the worst case scenario--current transfer only at the ends of each coil section--now results in a peak temperature above the allowed limit. A more accurate model is therefore needed to better establish the actual safety of the conductor design.

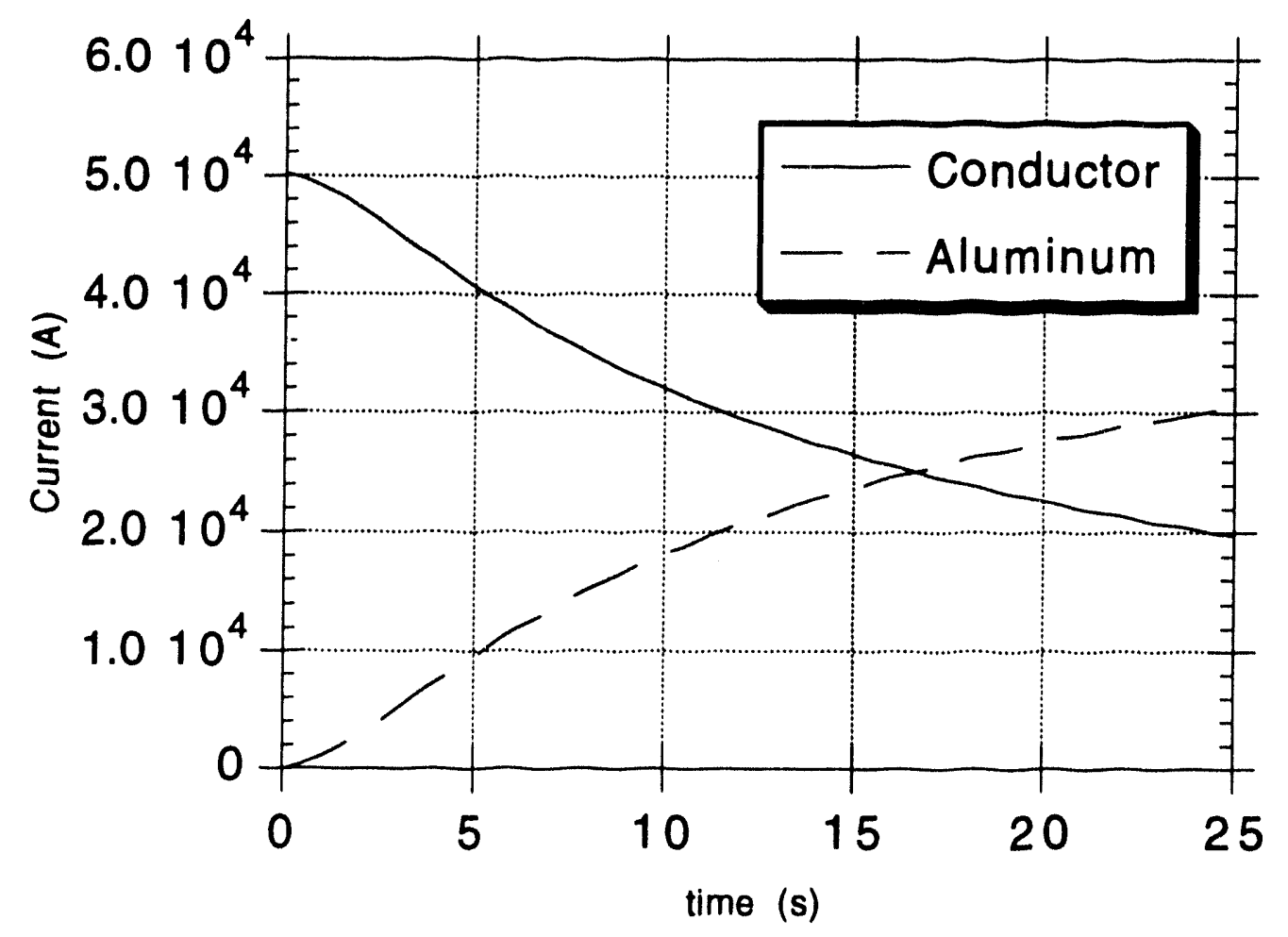

Figure 6. Currents in conductor and aluminum during quench with current transfer only at the ends of each coil section. Quench velocity $=20 \mathrm{~m} / \mathrm{s}$, equivalent inductance $=1 \mathrm{mH}$. No temperature effects are included. 


\section{CURRENT TRANSFER THROUGH CONDUIT}

In reality, the current transfer from the normal zone conductor to the external aluminum stabilizer occurs primarily through the stainless steel conduit and not at the ends of each coil section. Along the length of the conductor, the contact between the aluminum and superconductor is characterized by a transverse conductance per unit length, $g_{t}(\mathrm{mho} / \mathrm{m})$. The value of $\mathrm{gt}_{\mathrm{t}}$ is a measure of the resistance to current flow across the stainless steel conduit that separates the conductor from the aluminum. Besides the resistivity of the steel itself, the transverse conductivity must include the effects of contact resistance at both the steel-conductor and steel-aluminum interfaces. An estimate for the value of the transverse conductance per length will be given below (Equation 11).

To account for the effects of transverse conductance on the safety considerations of the GEM conductor, a model is first presented and then solved, making approximations as necessary. As will be seen, transverse conductance in the GEM conductor allows rapid current transfer out of the normal zone and thus contributes greatly to the safety of the design during quench.

\subsection{Current Transfer Model for the GEM Conductor including Transverse Conductance}

To accurately model the current transfer between the conductor and the aluminum sheath on short time scales, it is necessary to study the GEM conductor as a continuous length of differential circuit elements. Both inductive and transverse conductive effects have to be included in each element. Fig. 7 shows one differential circuit element of the GEM magnet with all resistances and inductances ( $r$ 's, l's, $\mathrm{m}$ ) given on a per unit length basis. Multiplied by the distance, $d x$, they represent the differential values in units of ohms and henries. The transverse resistance is likewise given as $1 /\left(g_{t} d x\right)$ ohms. The transverse current, $i_{t}$, is also given on a per unit length basis and multiplying by $\mathrm{dx}$ gives the differential value in units of amps. 
Using Kirchoff's voltage law, the following equation is obtained:

$$
\frac{1}{g} \frac{d i_{t}}{d x}+r_{c} I_{c}-r_{a} I_{a}+\left(\ell_{c}-m\right) \dot{I}_{c}-\left(\ell_{a}-m\right) \dot{I}_{a}=0
$$

where $i_{t}$ is transverse current per meter, $I_{c}$ is current in the conductor, and $I_{a}$ is current in the aluminum. Subscript " $c$ " refers to the properties of the composite (superconductor plus matrix) and subscript "a" refers to aluminum properties. If the total current in the conductor, $I_{0}$, is considered to be approximately constant on the short time scales being considered here (a conservative estiniate), then conservation of current gives:

$$
\begin{aligned}
& I_{c}+I_{a}=I_{0} \\
& i_{t}=-\frac{d I_{c}}{d x}=\frac{d I_{a}}{d x}
\end{aligned}
$$

The result is a second order partial differential equation for the current in the conductor:

$$
\frac{d^{2} I_{c}}{d x^{2}}-g \ell_{e q} \dot{I}_{c}-g\left(r_{a}+r_{c}\right) I_{c}=-g r_{a} I_{0}
$$

where $\ell_{\mathrm{eq}}=\imath_{\mathrm{a}}+\ell_{\mathrm{c}}-2 \mathrm{~m}$, the effective inductance per unit length.

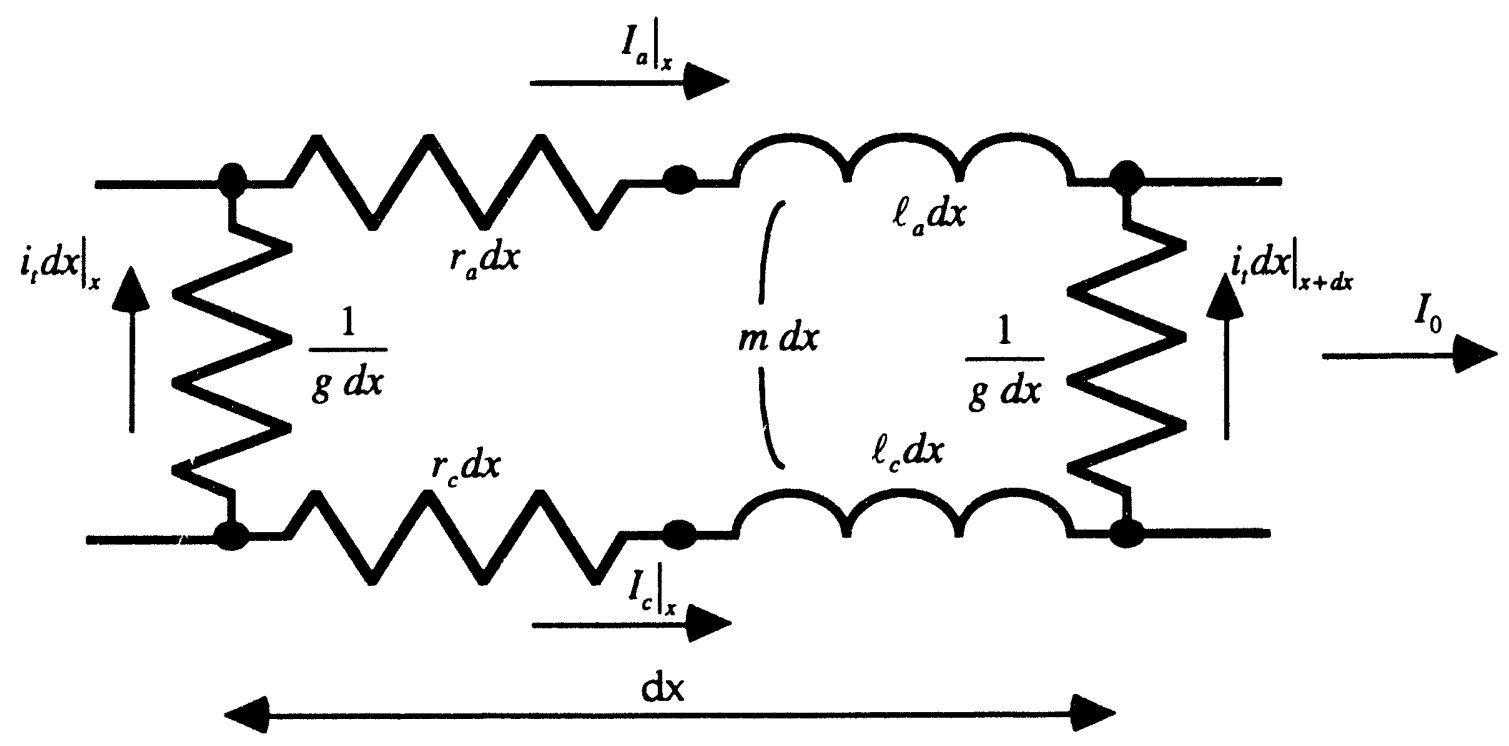

Figure 7. Differential element of the GEM conductor with resistances, inductances and currents. 


\subsection{The Constant Resistance Approximation to Transverse Current Transfer}

Equation (8) is a valid expression for the current in the conductor as a function of time and space. Unfortunately, in studying the GEM conductor during quench, equation (8) cannot be solved analytically because the resistances will not be constant in either time or space. To gain an understanding of the problem, however, the GEM conductor is first analyzed using the assumption that resistances are constant in time. The goal of this very conservative, simplified analysis is to determine if a more detailed investigation is necessary.

\subsubsection{Analytic Solution to the Constant Resistance Approximation}

As a first step in finding an approximate solution for the current in the conductor as a function of time and space, the GEM conductor is divided into two distinct regions: a normal zone region and a superconducting region. The location of the boundary between the two regions and the resistances of each region are considered to be constant. The problem is symmetric about the center of the normal zone, as diagrammed in Fig. 8.

With these assumptions, equation (8) can be solved analytically. Using Laplace transforms and appropriate boundary conditions at the center, the endpoints, and the interface, the equation can be solved in closed form in transform space for each of the two regions (with initial condition $\left.I_{C}(x, t=0)=I_{0}\right)$. The resulting equations are:

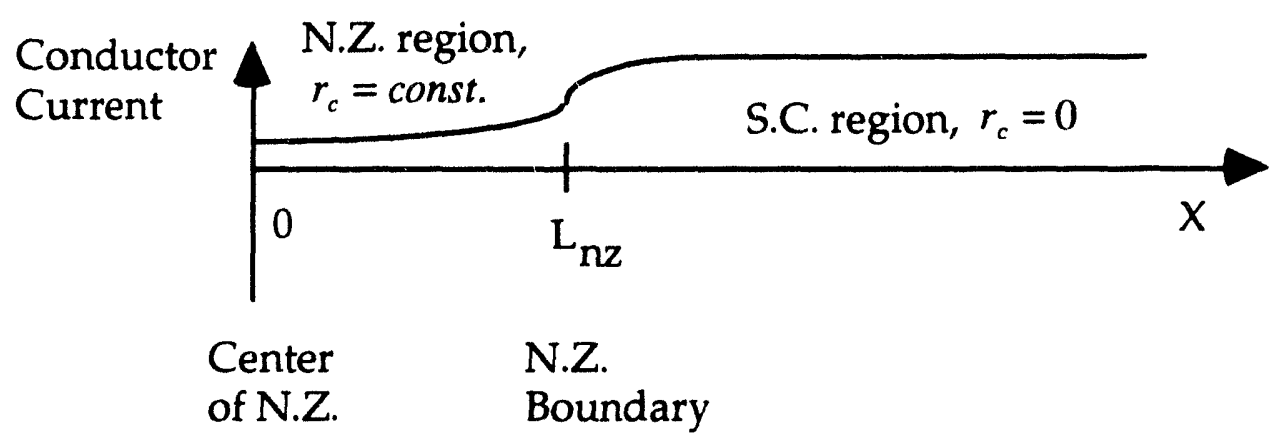

Figure 8. Diagram of the two domain, constant resistance approximation of a quench in the GEM Magnet conductor. 
in the normal zone $\left(0<\mathrm{x}<\mathrm{L}_{\mathrm{nz}}\right)$

$$
\begin{aligned}
& \hat{I}_{c}=I_{0}\left(\ell_{e q}+\frac{r_{a}}{s}\right)\left\{\frac{1}{\left(\ell_{e q} s+r_{a}\right)}+\frac{\alpha_{n z}}{\beta_{n z}}\right\} \\
& \alpha_{n z}=\left(\frac{1}{\left(\ell_{e q} s+r_{a}\right)}-\frac{1}{\left(\ell_{e q} s+r_{a}+r_{c}\right)}\right) \cosh \left(x \sqrt{g\left(\ell_{e q} s+r_{a}+r_{c}\right)}\right) \\
& \beta_{n z}=\cosh \left(L_{n z} \sqrt{g\left(\ell_{e q} s+r_{a}+r_{c}\right)}\right)+\sqrt{\frac{\left(\ell_{e q} s+r_{a}+r_{c}\right)}{\left(\ell_{e q} s+r_{a}\right)}} \sinh \left(L_{n z} \sqrt{g\left(\ell_{e q} s+r_{a}+r_{c}\right)}\right)
\end{aligned}
$$

and in the superconducting zone $\left(x>\mathrm{L}_{\mathrm{nz}}\right)$

$$
\begin{aligned}
& \hat{l}_{c}=I_{0}\left(\ell_{e q}+\frac{r_{a}}{s}\right)\left\{\frac{1}{\left(\ell_{e q} s+r_{a}\right)}-\frac{\alpha_{s c}}{\beta_{s c}}\right\}
\end{aligned}
$$

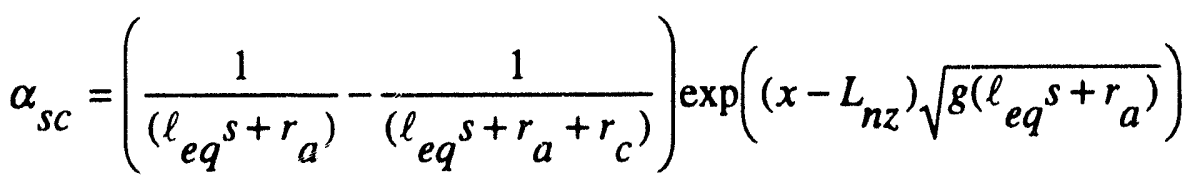

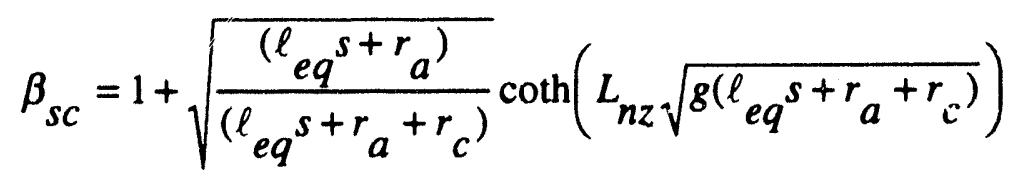

where the caret, " $\wedge, "$ indicates a Laplace transform and $s$ is the transform variable. Although the inverse transforms cannot be found in closed form, numerical integration over the complex plane gives highly accurate results for the currents as a function of time and space.

\subsubsection{Results of Constant Resistance Approximation for GEM Conductor}

For typical values of the GEM conductor, using a fixed $10 \mathrm{~m}$ normal zone (temperature of conductor $=10 \mathrm{~K}$ ) and a superconducting region with temperature $=4.5 \mathrm{~K}$, the resulting time development of the current profiles is shown in Fig. 9. The conductor current at the center of the normal zone falls toward a steady state value at a rate limited by inductive effects. Near the interface between the normal zone and superconducting region, current begins to flow from the aluminum sheath, through the stainless steel conduit, and back into the superconductor. 
Because the conductor resistance is held constant at a value corresponding to a temperature of $10 \mathrm{~K}$, the steady state current in the conductor hotspot $(\sim 25 \mathrm{kA})$ is drastically overestimated in this preliminary calculation. The joule heating associated with this current would bring the conductor over the prescribed $100 \mathrm{~K}$ safety limit in less than 3 seconds, as can be determined from Fig. 2. Ignoring other factors, this result would be unacceptable.

In actuality, however, as the conductor heats up due to joule heating, its resistance increases and the current in the conductor decreases proportionally. Fig. 10 plots the resistance per unit length of the conductor as a function of temperature. If the aluminum sheath is assumed to remain relatively cool on the short time scales considered here, its resistance per whit

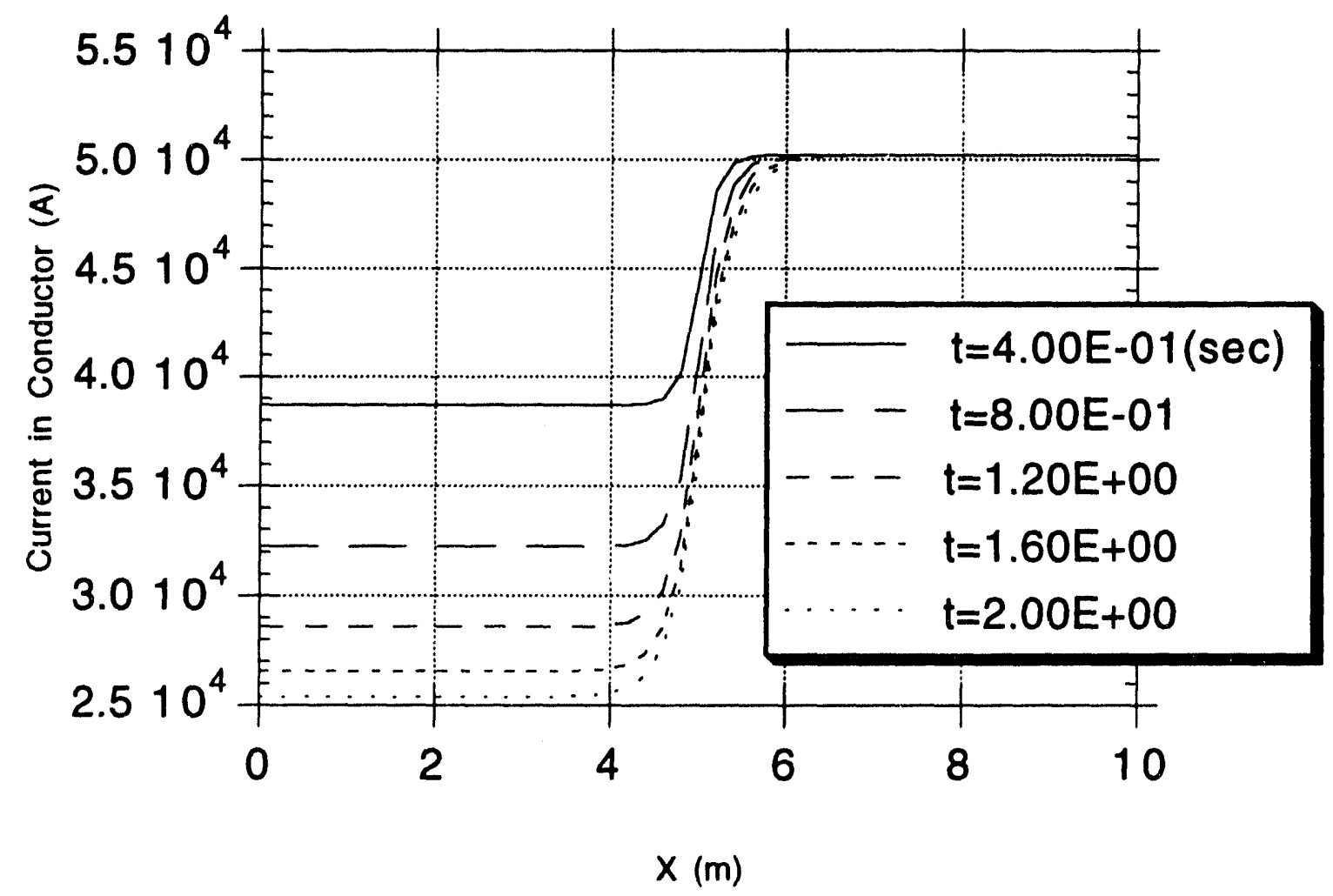

Figure 9. Time development of current profile in the GEM conductor for the constant resistance approximation. The normal zone extends over the region $-5 \mathrm{~m}<\mathrm{x}<5 \mathrm{~m}$. 
length is $\sim 1 \times 10^{-6} \mathrm{ohm} / \mathrm{m}$. As the conductor temperature rises, the resistance of the conductor is seen to be much higher than the resistance of the aluminum. As heating progresses, therefore, an increasing amount of current will transfer from the conductor to the sheath and thus further heating will be limited. This effect needs to be included to correctly judge the safety of the GEM conductor and for this reason a more accurate approximation of the quench problem is discussed in section 4.3.

\subsubsection{Validation of Model by Energy Minimization Techniques}

Before developing a more complicated model, it is useful to validate the simpler model presented above. Steady state current profiles can also be found using a minimization of energy technique. The total joule heating dissipated in the conductor, sheath, and conduit will reach a minimum when the currents reach their final values. Using a multi-variable function minimization routine, we obtain results which do indeed verify the asymptotically approached steady state predictions of equations (9) and (10) for the same two domain problem with constant resistances. Some thought was given to using a similar minimization technique to verify the transient (intermediate) solutions of the above model, but this avenue was not pursued.

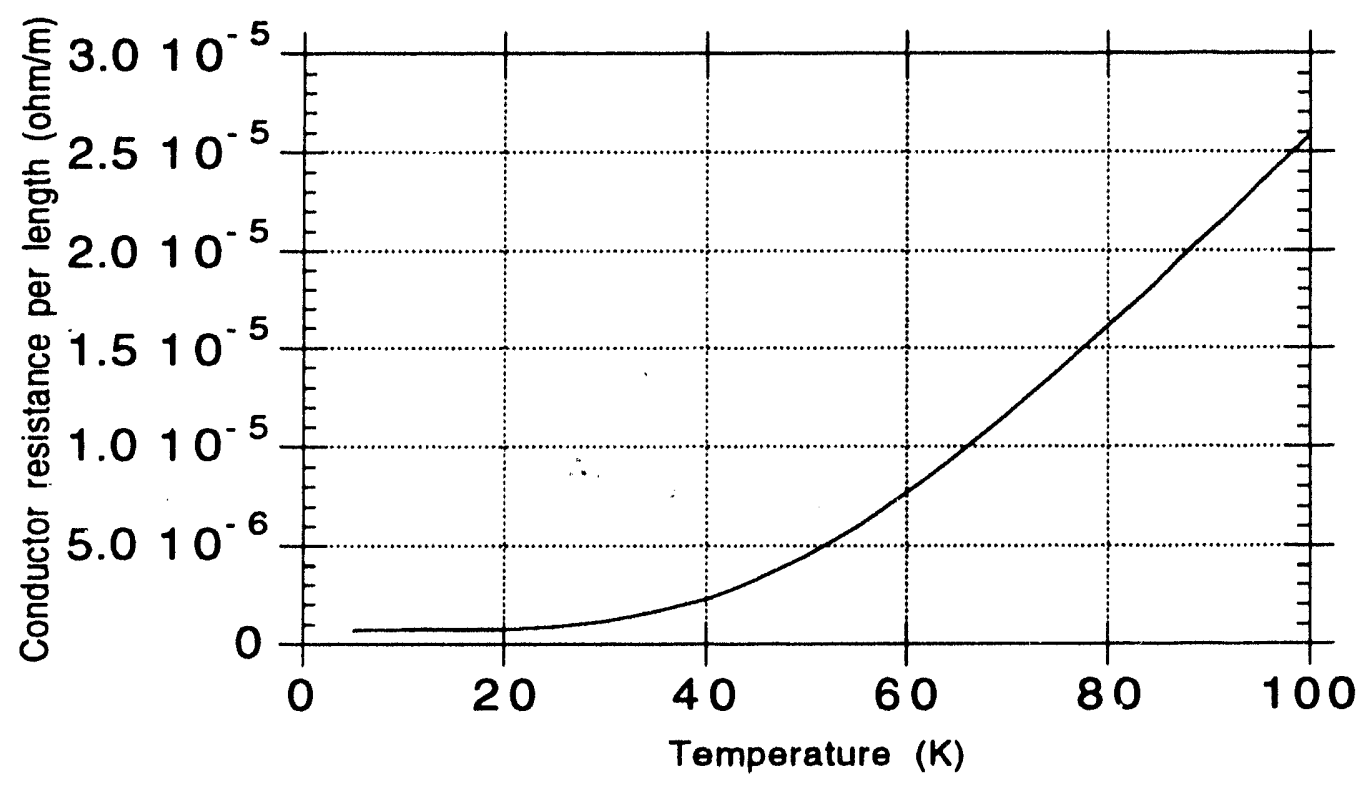

Figure 10. Conductor resistance per length vs. temperature for GEM magnet. The aluminum sheath is modeled as having a constant resistance per length of $\sim 1 \times 10^{-6} \mathrm{ohm} / \mathrm{m}$ 


\subsection{A Variable Resistance Approximation to Transverse Current Transfer}

In the model developed above, the current in the conductor is determined by equation (8). For the two domain assumption using constant resistances, the solution is given in equations (9) and (10). In the real case where the resistances along the length of the conductur are allowed to vary with temperature, however, the coefficients of equation (8) are not constant and a closed form solution cannot be found. The technique used to obtain estimates of the solution for the real case will now be discussed. Also, since the exact values for transverse conductance per length, $g_{t}$, and equivalent inductance per length, $\ell_{\mathbf{e q}}$, are not known, the sensitivity of the model to these parameters will be determined.

\subsubsection{An Approximate Solution to the Current Transfer Equation for Varying Resistances}

To find an approximate solution, the GEM conductor is again divided into a fixed normal zone region and a superconducting region. However, the properties of the normal zone region are now allowed to change with time. To accomplish this, the model is run in small time steps and equations (9) and (10) are inverse transformed using new properties at each step. The approximations needed are:

1.) within each domain (normal zone and superconducting), the values of resistance and heat capacity are constant in space;

2.) the initial condition for each time step is the solution of the previous time step;

3.) the interface boundary condition is simplified by neglecting terms arising from the space-derivative of the initial condition;

4.) the model is adiabatic (no convection, no conduction).

The resulting equations for the Laplace transform of the current in the conductor are identical to equations (9) and (10) except the initial current, $I_{0}$, is now a function of $x$, the distance from the center of the normal zone. The actual current is calculated by first evaluating the temperature dependent resistance in the normal zone and then integrating equations (9) and (10) over 
one timestep. Using the resulting value of the current in the conductor at the center of the normal zone, the joule heating adiabatically absorbed in the conductor is found and a new normal zone temperature is calculated. This procedure is repeated for each timestep. With this technique, the effects of the rising resistance of the normal zone can be studied.

To ensure a better approximation, a "dynamic" timestep size is used. For each step, the size is determined either by a maximum allowable temperature rise per step (usually $1 / 2-1 \mathrm{~K}$ ) or a fixed maximum timestep (usually $0.1 \mathrm{~s}$ ), whichever is smaller.

The effects of neglecting a term in the equation for the current in the conductor--assumption (3)--above, were studied for a test case in which the resistances were artificially held constant. The results of this test were compared to the analytic solutions available from equations (9) and (10). Although the assumption did lead to errors in the overall shape of the current profile at each timestep, the current at the center of the normal zone was not greatly affected. The assumption is therefore allowable since it is only the hotspot temperature which determines the safety of the conductor.

If a better approximation were necessary, a finite difference scheme would be a better way to approach the model. However, the Laplace transform method is used here since it requires only a modification of a simpler model already developed and the results show that greater accuracy is unnecessary.

\subsubsection{The Effects of Transverse Conductivity}

The transverse conductance per unit length across the stainless steel, $g_{t}$, includes the resistivity of the steel as well as the contact resistance at both the conductor-steel and steel-aluminum interfaces. For the purposes of this study, a nominal value of $g_{t}$ was obtained by using $1 / 5$ the conductance of the stainless steel itself:

$$
g_{t} \approx \frac{1}{5} \frac{P}{\rho_{s s} t}
$$

The factor of 5 approximates the effects of contact resistivity; $P$ is the perimeter of the conduit; $\rho_{\mathrm{ss}}$ is the stainless steel electrical resistivity 
( $5 \times 10^{-7} \mathrm{ohm}-\mathrm{m}$ at $\left.5 \mathrm{~K}\right)$; and $\mathrm{t}$ is the conduit thickness. For the GEM conductor, this value of $g_{t}$ is $\sim 1 \times 10^{7} \mathrm{mho} / \mathrm{m}$.

The results in Fig. $11(a, b$, and $c)$ show the development in time of the current profiles as well as the hotspot temperatures for several values of transverse conductance, $\mathrm{gt}_{\mathrm{t}}$. In each case a static normal zone of $10 \mathrm{~m}$ length is used with the center of the normal zone shown at $x=0$. Each case uses a conservative estimate of the equivalent inductance, $\ell_{\mathrm{eq}}=1 \times 10^{-6} \mathrm{H} / \mathrm{m}$, determined $b y$ dividing the inductance calculated earlier for the entire coil section (equaisior. 10) by its length.

From the figures, it can be seen that for the chosen values of transverse conductance, current transfer out of the superconductor takes place within a few meters of the normal zone boundary. As long as the half-normal zone length is greater than the current transfer length, the current in the conductor at the center of the normal zone is independent of transverse conductance. That is, the current profile reaches an asymptotic value at a point significantly removed from the center of the normal zone. Thus, with the rapid growth rate of a normal zone in the GEM conductor $(20 \mathrm{~m} / \mathrm{s})$ and the relatively short transfer length $\left(\sim 5 \mathrm{~m}\right.$ for a very conservative $\mathrm{g}_{\mathrm{t}}$ of $\left.1 \times 10^{5} \mathrm{mho} / \mathrm{m}\right)$, the joule heating at the hotspot can be considered independent of $g_{t}$ over a wide range. The relative insensitivity of the hotspot temperature to the transverse conductance precludes the need for a more exact estimate of $g_{t}$ for the GEM conductor. 
Curr. In Cond. ; gt $=1 \mathrm{e} 7 \mathrm{mho} / \mathrm{m}$

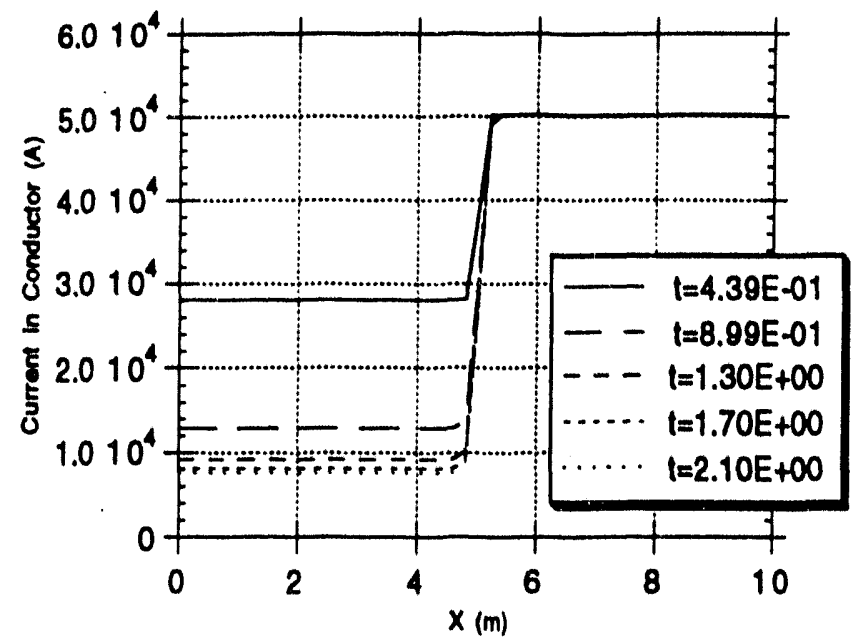

Curr. in Cond. ; gt $=106 \mathrm{mho} / \mathrm{m}$
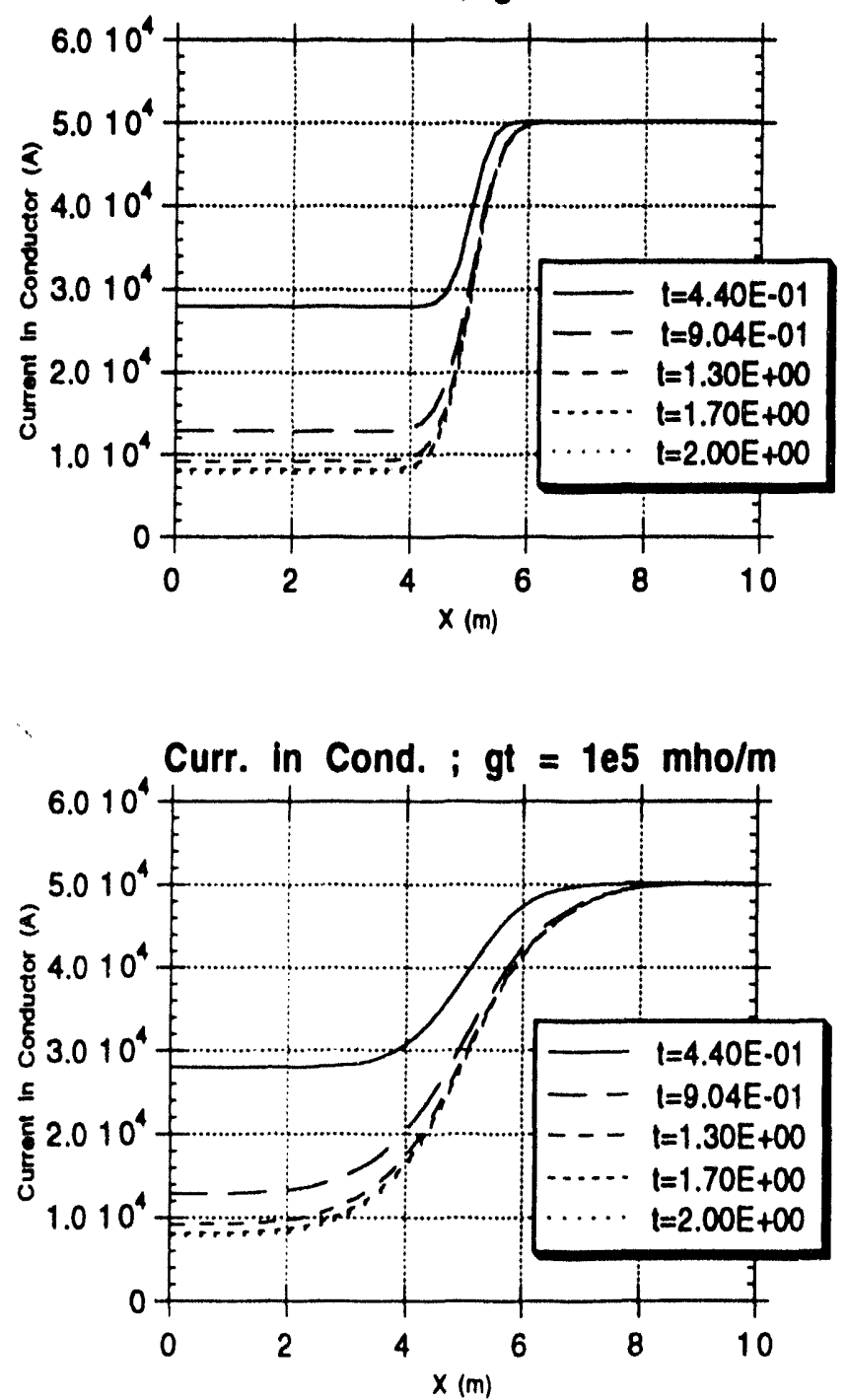

(a)

Temp. at Hotspot ; gt $=107 \mathrm{mho} / \mathrm{m}$

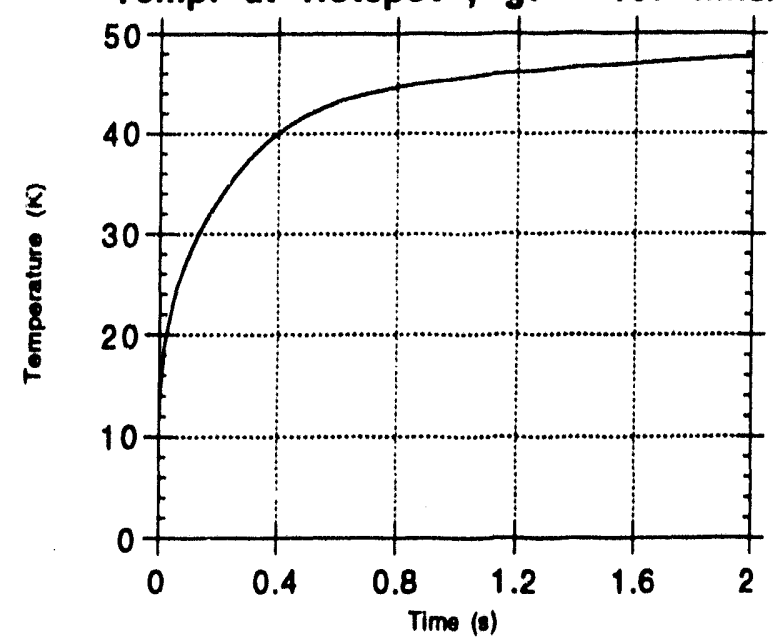

Temp. at Hotspot ; gt $=106 \mathrm{mho} / \mathrm{m}$

(b)

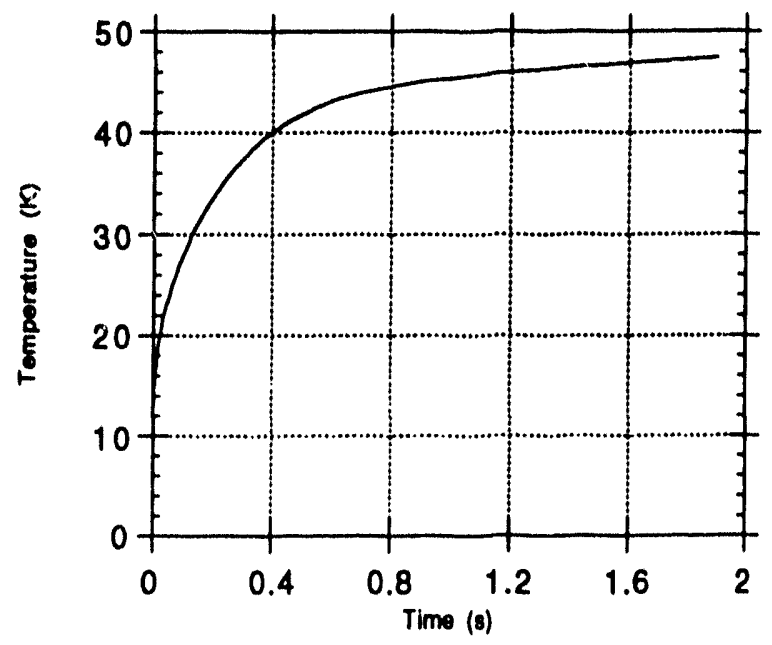

Temp. at Hotspot; gt $=1$ e5 $\mathrm{mho} / \mathrm{m}$

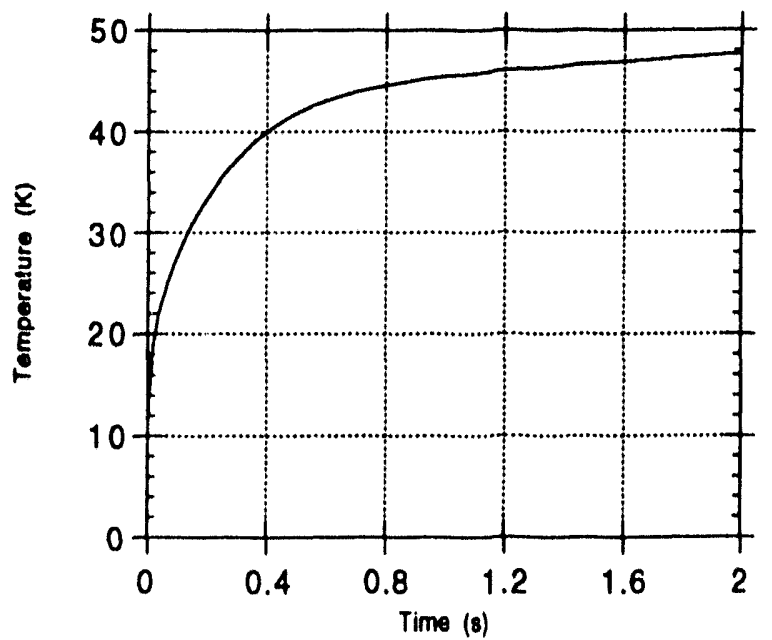

Figure 11 a,b,c. Current profiles vs. time and Hotspot temperatures vs. time for a static normal zone $(-5<x<5 \mathrm{~m})$ and differing transverse conductances, gt. (Equivalent inductance $=1 \mathrm{micro}-\mathrm{Henry} / \mathrm{m}$ ) 


\subsubsection{The Effects of Equivalent Inductance}

Because the safety of the conductor is determined by the temperature response in the first few seconds, it is important to work with a model which includes the important transient effects. To demonstrate the motivation for including these effects in the transfer conductance model, Fig. 12 shows a characteristic time development of the current in the conductor at the center of a static $10 \mathrm{~m}$ normal zone for three different scenarios. In scenario $\mathrm{A}$, the resistance of the normal zone is kept constant despite heating. In scenario $B$, the resistance varies as the temperature increases due to joule heating, but still no inductive effects are included. In scenario $C$, inductive effects as well as variable resistances are included. Each result uses an estimated transverse conductance per length of $\mathrm{g}_{\mathrm{t}}=1 \times 10^{6} \mathrm{mho} / \mathrm{m}$, and the results of scenario $\mathrm{C}$ are shown for equivalent inductances of both $\ell_{\mathrm{eq}}=1 \times 10^{-6}$ and $\ell_{\mathrm{eq}}=1 \times 10^{-5}$ $\mathrm{H} / \mathrm{m}$.

A priori, looking only at the current profiles for each scenario, it is possible to say:

1.) Scenario $\mathrm{A}$ (constant resistance) yields higher temperatures than scenario B (variable resistance) because the resistance of the superconductor increases with temperature and heating is proportional to $1 /$ resistance $(\mathrm{Q} \sim \mathrm{R} \mathrm{I} 2, \mathrm{I} \sim 1 / \mathrm{R}-\mathrm{Q} \sim \mathrm{Q} \sim 1 / \mathrm{R})$.

2.) Scenario $C$ (variable resistance + inductance) yields higher temperatures than scenario $B$ (variable resistance) since inductive effects slow the transfer of current (and thus prolong heating) out of the conductor.

3.) On short enough time scales, scenario $C$ (var. resistance + inductance) yields higher temperatures than scenario $\mathrm{A}$ (const. resistance) for reasons similar to 2.), above. On long enough time scales, scenario A produces higher temperatures than scenario $\mathrm{C}$ since the current in the conductor remains higher.

The resultant temperature profiles from these three scenarios, generated using the same values of $g_{t}$ and $\ell_{\mathrm{eq}}$, are given in Fig. 13. The curves show the temperature rise in the conductor at the hotspot due to adiabatic joule heating from the conductor current. From the figure, it is evicient that on a 


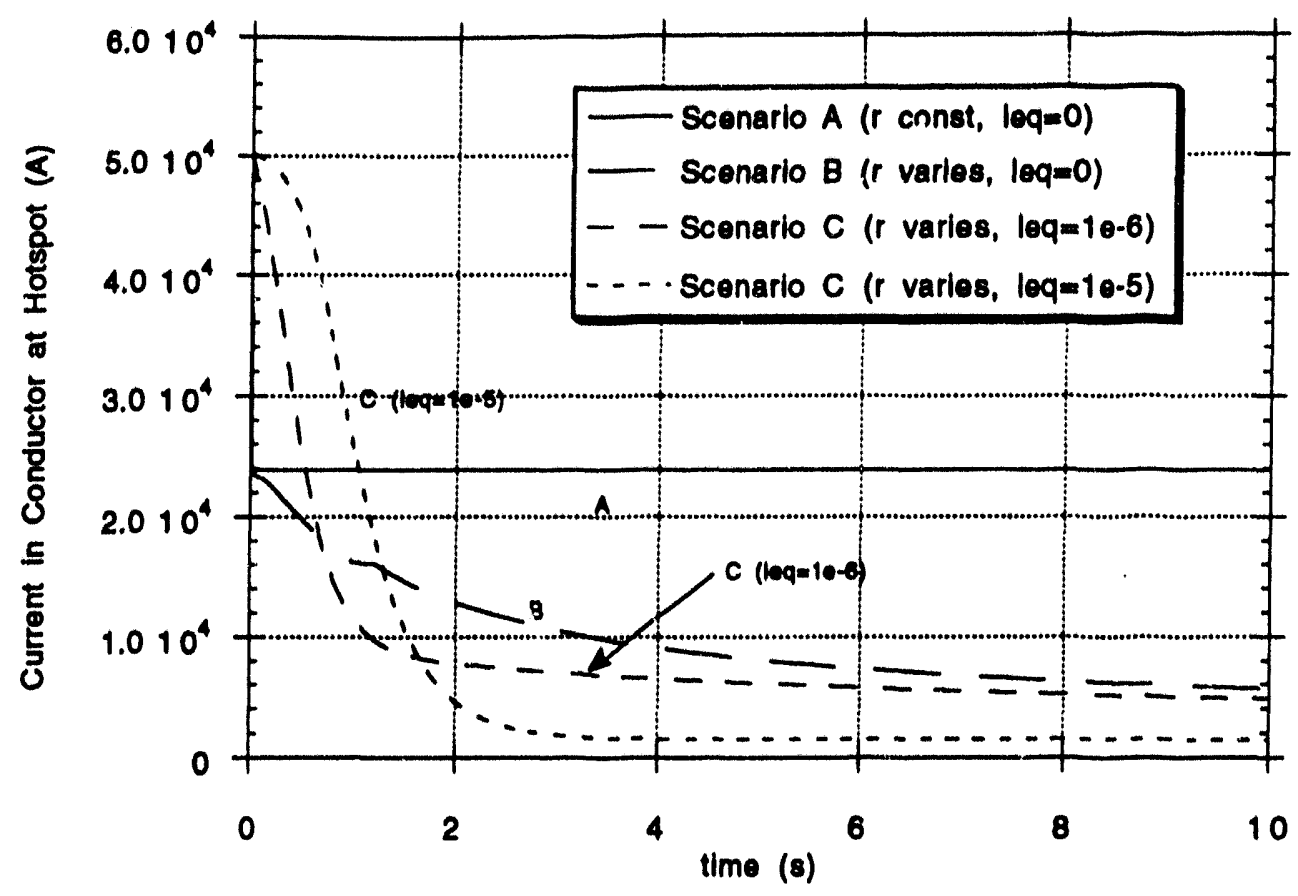

Figure 12. Current in the Conductor v8. time for three different models.

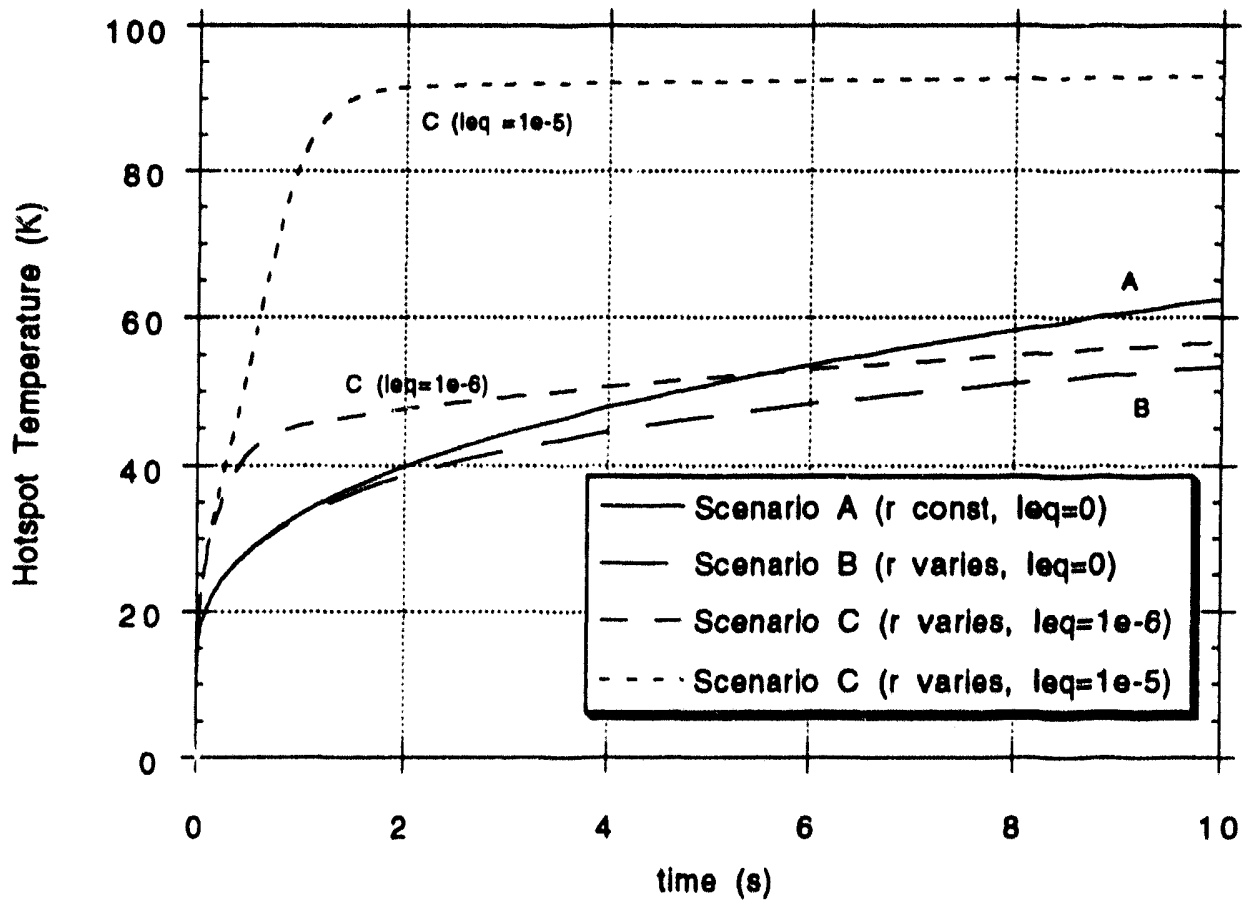

Figure 13. Hotspot Temperature vs. Time for three different current sharing models. 
time scale of a few seconds, inductive effects play a large role in determining the peak temperature in the conductor. While models which do not include inductance might be used for studying long term protection issues, short-term safety analysis requires an inductive model.

During this study, a conservative estimate of $\ell_{\mathrm{eq}}=1 \times 10^{-6} \mathrm{H} / \mathrm{m}$ is used as a nominal value. Using this value, the peak conductor temperature begins to level off at $\sim 50 \mathrm{~K}$ after $\sim 1$ second, as shown in Fig. 13. To study the sensitivity of peak temperature to the inductance, increasing the already conservative estimate by 2 factor of $10\left(\ell_{\mathrm{eq}}=1 \times 10^{-5} \mathrm{H} / \mathrm{m}\right)$ gives a peak conductor temperature of $\sim 90 \mathrm{~K}$, leveling off at $\sim 2$ seconds, also shown in Fig. 13. Even this result is acceptable in terms of allowed peak conductor temperature which suggests that the safety of the magnet is not dependent on accurate estimates of the inductance.

\section{CONCLUSIONS}

The GEM Detector magnet meets the safety requirement of $100 \mathrm{~K}$ maximum temperature during quench. Using conservative estimates of transverse conductance per length $\left(g_{t}=1 \times 10^{7} \mathrm{mho} / \mathrm{m}\right)$ and equivalent inductance per length $\left(\ell_{\mathrm{eq}}=1 \times 10^{-6} \mathrm{H} / \mathrm{m}\right)$, the peak temperature in the GEM conductor model reaches $\sim 50 \mathrm{~K}$ two seconds after initiation of quench. This result neglects the cooling effects of conduction and convection to the helium and is therefore very conservative. At later times, the temperature only rises slowly, even in the absence of a protection circuit that would reduce the overall energy dissipated in the magnet. These results can be seen in Fig. 13. Thus, the magnet can be considered safe at both short and long time scales.

The inherent safety of the conductor can be considered a result of the transverse conductance. The short current transfer lengths calculated for reasonable estimates of $\mathrm{g}_{\mathrm{t}}$ minimize inductive effects and allow the current in the conductor to drop more quickly than if transfer occurred only at the ends. With a quick transfer of the current out of the conductor, joule heating is reduced and the adiabatic hotspot temperature remains low. The conductor need not rely on a "quenchback effect" to be considered a safe design. 


\section{FUTURE WORK}

The model currently in use is good enough to determine with confidence that the temperature response of the GEM magnet during quench will remain within allowed limits. However, the solution of the full model under the dynamic conditions of a quench uses certain simplifications. While the necessary approximations do not make the general results of the model unreliable, they do affect the accuracy of the results. For this reason, the model is currently being improved to eliminate the need for the simplifying assumptions.

With the improved model, the following effects might also be incorporated: a quench zone growing at an adiabatically determined quench velocity, joule heating in the aluminum and conduit due to axial and transverse currents, and axial heat conduction. Another factor which could also be included in the analysis is the electrical diffusivity of the relatively large sheath conductive path. Estimates of the diffusion time constant for the aluminum are on the order of 50 to 100 milli-seconds. Making these improvements is not expected to alter the general conclusion that the GEM magnet behaves safely with respect to temperature rise in the conductor, but it will provide for a better ability to study future conductor designs.

\section{ACKNOWLEDGMENTS}

The authors are grateful to Dr. S.S. Shen for helpful discussions and useful comments.

This work was performed under the auspices of the U.S. Department of Energy by the Lawrence Livermore National Laboratory under Contract W-7405-ENG-48.

This research was performed under appointment to the Magnetic Fusion Energy Technology Fellowship Program administered by Oak Ridge Institute for Science and Education for the U.S. Department of Energy. 


\section{REFERENCES}

1. J.V. Minervini, P.G. Marston, et al., "Conductor Design for the GEM Detector Magnet," presented at the Fifth Annual International Symposium on the Super Collider, May 6-8, 1993, San Francisco, CA.

2. E.A. Chaniotakis, "Quench Simulations of the GEM Coil," Memorandum to GEM Group, Reference \# GEM/05-06-93/EAC, May 6, 1993, Massachusetts Institute of Technology, Cambridge, MA.

3. J.R. Hale, "Current Transfer form Cable to Sheath During Quenching," Memorandum to GEM Conductor Development Group, Reference \# MIT-GEM-EM-21, June 30, 1993, Massachusetts Institute of Technology, Cambridge, MA.

4. M.N. Wilson, Superconducting Magnets, 1990, Oxford University Press, New York.

5. L. Bottura, Various Fortran Routines, NET Team, July 24, 1990.

6. W.V. Hassenzahl, "Heat capacity of Nb-Ti," private communication, July, 1993, Lawrence Livermore National Laboratory. 

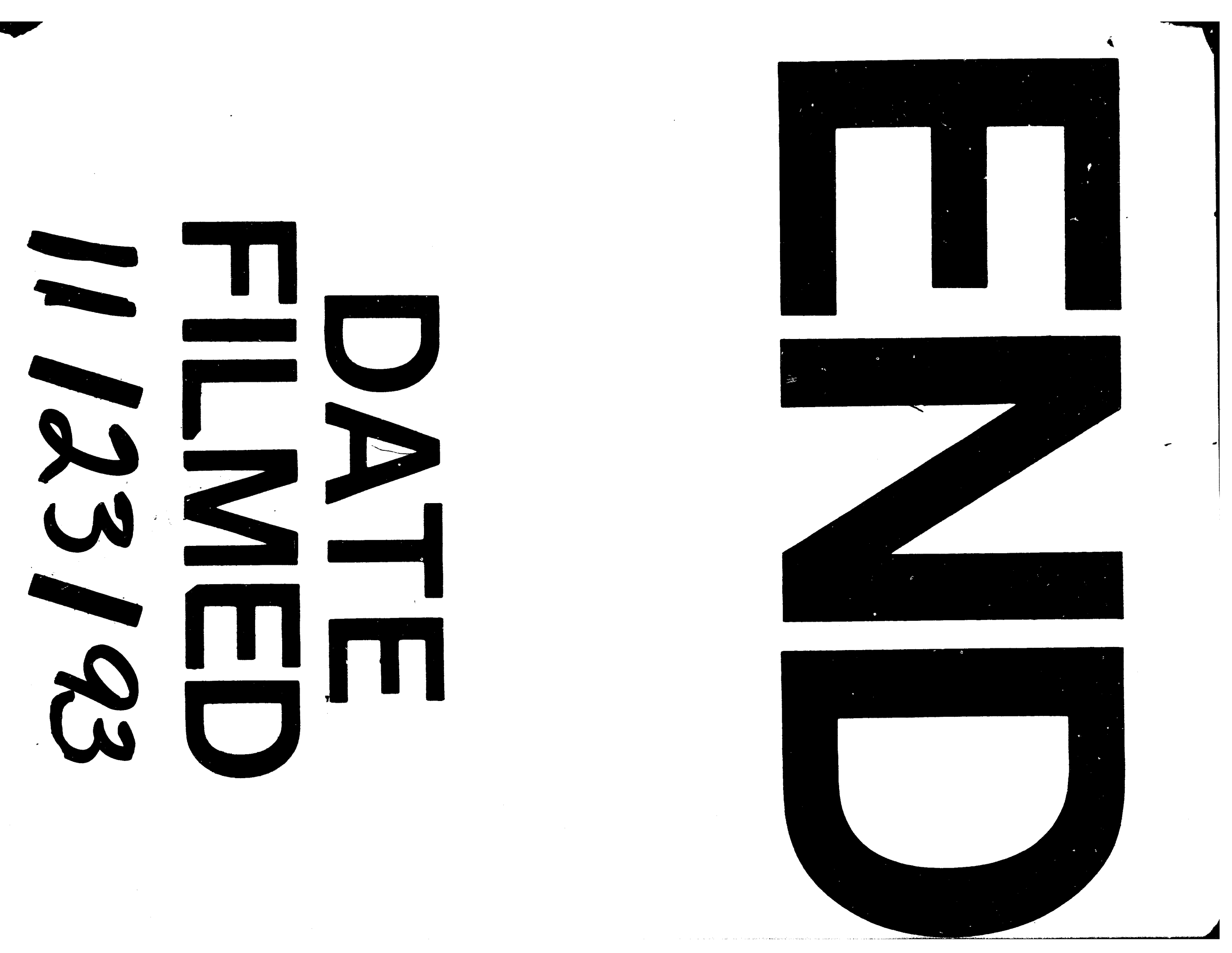


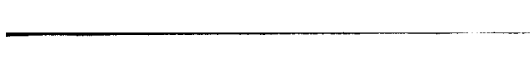

$\longrightarrow$

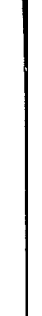

